\title{
Assessment of Haemodynamic Remodeling in Fetal Aortic Coarctation using a Lumped Model of the Circulation
}

\author{
Paula Giménez Mínguez ${ }^{1 *}$, Bart Bijnens ${ }^{1}$, Gabriel Bernardino ${ }^{1}$, Eric Lluch ${ }^{2}$, Iris Soveral ${ }^{3}$, Olga Gómez ${ }^{3}$, \\ Patricia Garcia-Canadilla ${ }^{1}$ \\ ${ }^{1}$ Univesitat Pompeu Fabra, Barcelona, Spain; ${ }^{2}$ Philips Research Medisys, Suresnes, France; ${ }^{3}$ Fetal i+D \\ Fetal Medical Research Center, Barcelona, Spain \\ * paula.gimenez01@estudiant.upf.edu
}

\begin{abstract}
Introduction: Aortic coarctation is one of the most difficult cardiac defects to diagnose before birth, and it accounts for $8 \%$ of congenital heart diseases. Antenatal diagnosis is crucial for early treatment of the neonate and to decrease the risk of morbidity and mortality; however the fetal hemodynamic changes are not fully understood and current imaging methods are limited to accurately diagnosis this congenital defect. Objective: We propose to use a lumped model of the fetal circulation to provide insights into the hemodynamic changes in fetuses with aortic coarctation, and thus helping to improve its diagnosis. Methods: To achieve this goal a patient-specific lumped model of the fetal circulation was implemented in OpenCOR, including the modeling of different types and degrees of aortic coarctation. A parametric study of degree and type of coarctation was performed, where blood flow distribution, cerebroplacental ratio, pressure drop over the coarctation and left ventricular pressure were quantified. Results: Obvious changes in the fetal hemodynamics were observed only from $80 \%$ of coarctation, corresponding to the clinically used cutoff for pressure drop of $20 \mathrm{mmHg}$. Furthermore, the observed hemodynamic changes were different depending on the location and degree of the coarctation.
\end{abstract}

Keywords: fetal circulation, modeling, and aortic coarctation

\section{Introduction}

Aortic Coarctation accounts for around $8 \%$ of heart defects. This congenital disease consists on the narrowing of the distal aortic arch in mild cases; thus reducing the blood flow in the fetal aortic arch [1]. According to different studies $[2,3]$, hypoplasia of the whole aortic arch is also observed in most severe cases.

Coarctation of the aorta remains one of the cardiac defects more difficult to diagnose before birth. Prenatal diagnosis is of critical importance to improve survival and reduce morbidity [4]. In most severe cases, suspicion rises when there is ventricular disproportion, however; a slight degree of physiological disproportion appears during the third trimester of gestation [5]. Consequently, current echography methods lead to both, high false-positive and false-negative rate during diagnosis [4, 6]. Additionally, the hemodynamic remodeling induced by the disease is yet not fully understood, partly due to the wide variety of fetal aortic coarctation types and degrees of severity.

Accordingly, there is a need to develop new technologies that allow accurate prenatal diagnosis of aortic coarctation and that help clinicians to better understand the disease. Currently, in clinical practice ultrasonographic evaluation, including measurement of right and left ventricular size, isthmus and ductal diameters, is used to diagnose aortic coarctation. Still, these measurements have low sensitivity and specificity $[6,7]$. On the other hand, patient-specific lumped models of the fetal circulation have shown to be a great approach to understand the underlying mechanisms of fetal hemodynamics, both under healthy and pathological conditions, such as shown in Garcia-Canadilla et al. [8,9]. 
Thereby, our purpose is to re-implement the fetal circulation model proposed in [9] in CellML using OpenCOR, an open-source cross-platform modelling environment [10], which favors model reuse amongst researchers. Besides, the modular structure of CellML allows users to easily add/remove new components to the model for future applications. The model will be used to simulate fetal circulation subjected to aortic coarctation.

\section{Material and methods}

\subsection{Patient-Specific Model}

\section{Lumped Model of the Fetal Circulation}

The fetal circulation model [9] was implemented in OpenCOR (version 0.5) using the CellML language. The ascending aorta of the original model was split into 3 different arterial segments: ascending aorta, ascending and descending aortic arches as shown in Figure 1. Moreover, a resistor distal from the aortic-ductal junction was included, representing distal aortic coarctation. The length of the segment was fixed at a $10 \%$ of the total ascending aorta length, and the radius was set initially to be equal to the radius of the ascending aorta. These changes in the model made possible the simulation of a variety of aortic coarctation scenarios.

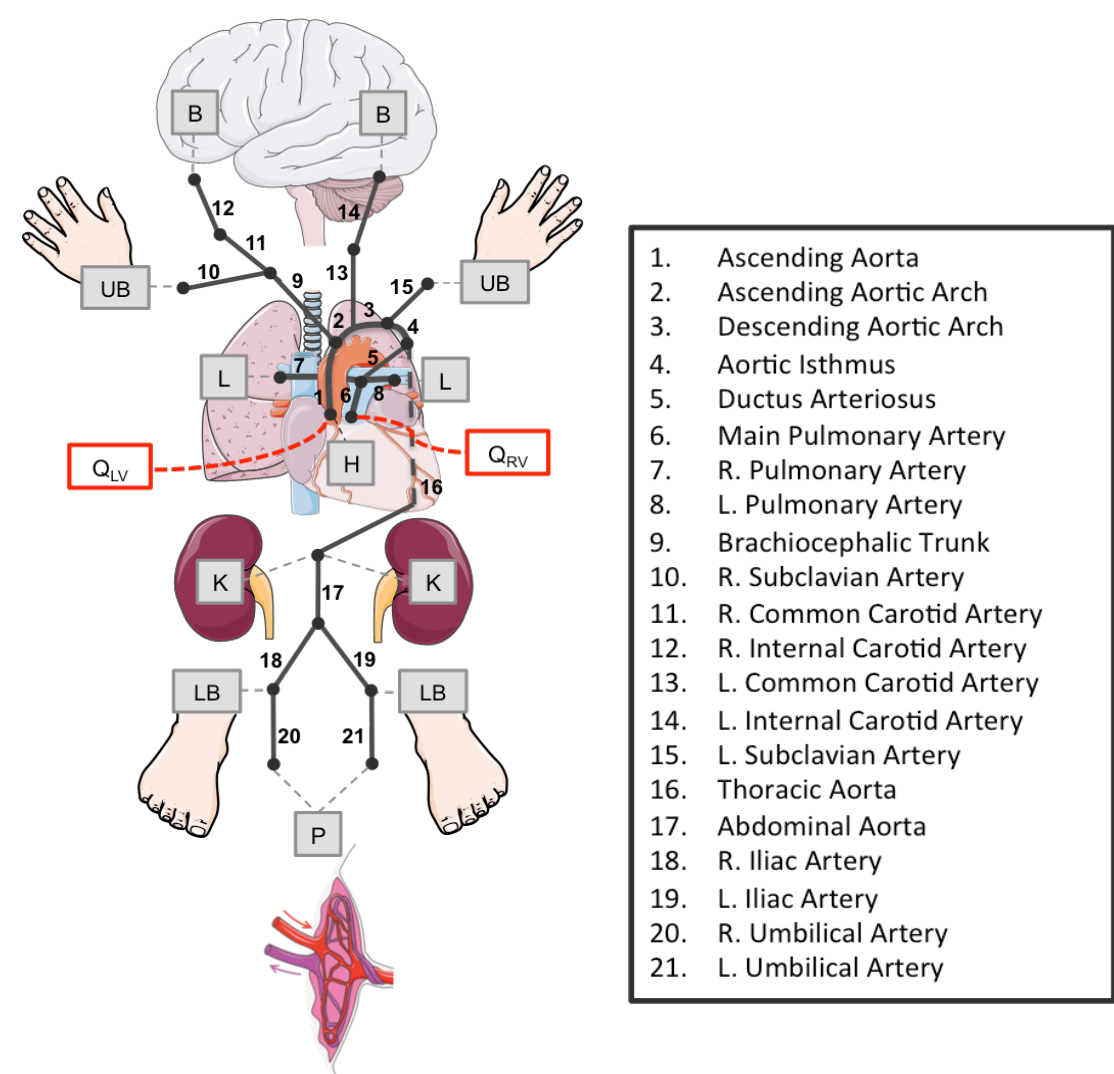

Fig 1. Lumped model scheme of the fetal circulation consisting on 21 artery segments (lines), and 12 vascular beds (boxes). Two blood flow inputs were considered: Left and right ventricular outflows. UB: upper body, B: brain, L: lungs, H: heart, K: kidneys, LB: lower body, P: Placenta. 
The equivalent lumped model circuit was built by interconnecting arterial segments and vascular beds. Each arterial segment contained a capacitor (C) representing arterial compliance, a resistor $(\mathrm{R})$ representing resistance to blood flow through the arterial segment, and an inductor (L) describing blood inertia. The vascular bed components consisted of a three-element Windkessel model, which included one resistor $\left(R_{c}\right)$, representing resistance of the characteristic impedance of the feeding artery, leading to a parallel circuit with a resistor $\left(\mathrm{R}_{\mathrm{p}}\right)$ and a capacitor $\left(\mathrm{C}_{\mathrm{p}}\right)$ describing peripheral resistance and compliance respectively.

Furthermore, CellML language was used to implement the improved version of the fetal circulation model, thanks to its modular structure that allow easily defining interconnected individual components (such as arterial segments and vascular beds), establishing connections between them, and importing the individual components into the model. Regarding the OpenCOR solver we used CVODE with a time step of 0.001 seconds.

\section{Patient-Specificity}

Patient-specific data were used to build the model, including: output flow from left $\left(Q_{L V}\right)$ and right $\left(Q_{R V}\right)$ ventricles, fetal gestational age $(G A)$ and the estimated fetal weight $(\mathrm{EFW})$. All the data were obtained from the same control fetus as used to validate the model described in [8].

All the electrical components of the equivalent circuit were fitted to a control healthy fetus of 33.28 weeks of GA and an EFW of $2250 \mathrm{gr}$. The dimensions of all arterial segments (radius, length and thickness), Young's Moduli, blood viscosity, and electrical components of all vascular beds $\left(\mathrm{R}_{\mathrm{p}}, \mathrm{C}_{\mathrm{p}}\right)$, were calculated according to the GA and EFW of the fetus, as described in [9]. Particularly, ascending aorta, and aortic arch new segments' dimensions were calculated based on [11].

\subsection{Modelling Aortic Coarctation}

Four different scenarios of aortic coarctation were considered in this study and are illustrated in Figure 2.

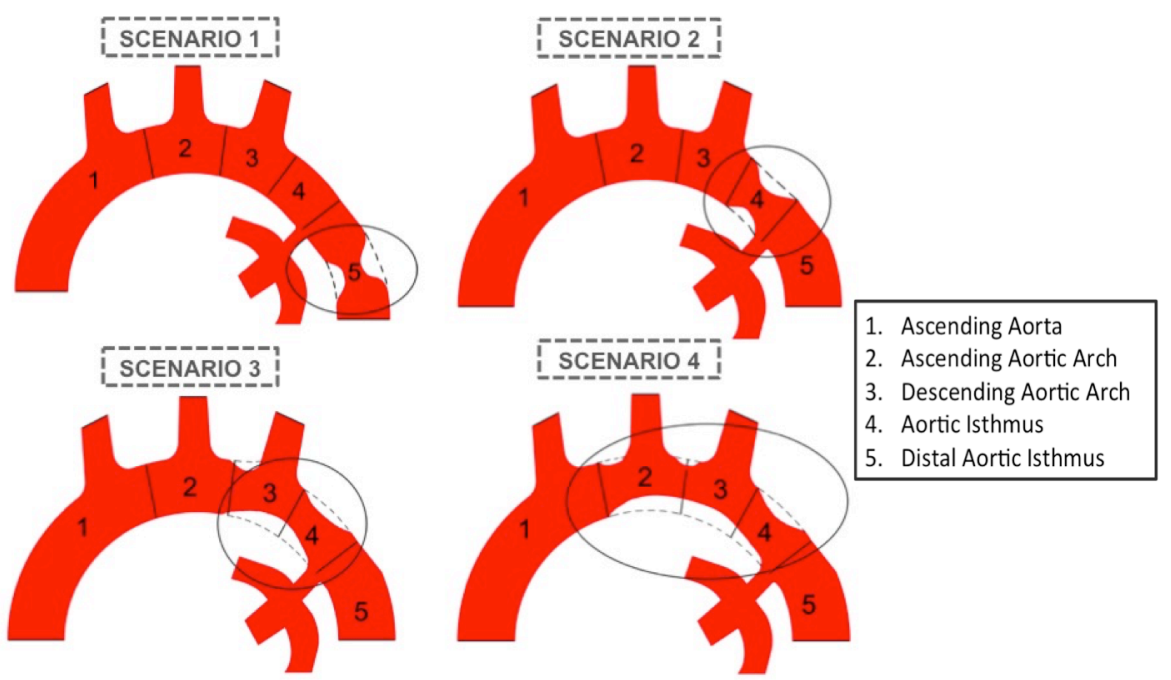

Fig 2. Aortic Coarctation types. Scenario 1: coarctation of distal aortic isthmus; Scenario 2: hypoplasia of aortic isthmus; Scenario 3: hypoplasia of aortic isthmus and descending arch; Scenario 4: hypoplasia of aortic isthmus, descending and ascending arches. 


\section{Modeling different scenarios}

To model the first scenario, a reduction in the radius of the aortic segment located distal from the aortic-ductal junction was introduced, simulating different degrees of coarctation ranging from $0 \%$ (normal conditions) to $90 \%$. Scenarios 2,3 and 4 where modeled by reducing the radius of the corresponding arterial segment/s denoted in Figure 2.

\section{Modeling ventricular disproportion in aortic arch hypoplasia}

In addition, ventricular disproportion was modeled in aortic arch hypoplasia (Scenario 4), by simulating different proportions of left (LV) and right ventricular (RV) outflows: $50-50 \%$ (no disproportion), $40-60 \%, 30-70 \%$ and $20-80 \%$, respectively.

\section{Parametric Study of Coarctation Degree}

The effect of aortic coarctation in the fetal circulation was analyzed by decreasing the radius of the different regions a given percentage. The following percentages of narrowing were considered: $0 \%$ (normal conditions), 50\%, 70\%, $80 \%$, and $90 \%$. Cerebro-placental ratio, pressure drop over the narrowed area and left ventricular pressure were measured for each scenario. Cerebro-placental ratio was computed as the maximum systolic brain flow over the maximum systolic placental flow. Pressure drop was calculated as the difference between the maximum input and the maximum output pressures of the narrowed area. Moreover, blood flow in the aortic isthmus (AoI), middle cerebral artery (MCA) and umbilical artery (UA) were also obtained. In the present model, MCA blood flow was defined as the $75 \%$ of the internal common carotid artery outflow.

\section{Results}

\subsection{Modeling different scenarios}

The AoI blood flow showed a progressive reversal as the percentage of coarctation increased in the distal aortic isthmus (Scenario 1, Figure 3A). In the rest of scenarios, blood flow in the AoI was reduced until it became $0 \mathrm{ml} / \mathrm{s}$ for a $100 \%$ of coarctation, as expected. It can be observed that no differences were notice until a $70 \%$ of stenosis was reached. Regarding MCA and UA blood flows (Figure 3B, $\mathrm{C})$, they increased and decreased respectively when increasing the amount of coarctation. The increment in MCA blood flow was larger in the most severe scenario corresponding to a tubular hypoplasia (Scenario 4), as well as in the case of coarctation distal to the aortic-ductal junction (Scenario 1); while reduction in UA blood flow was much more significant in Scenario 1. 

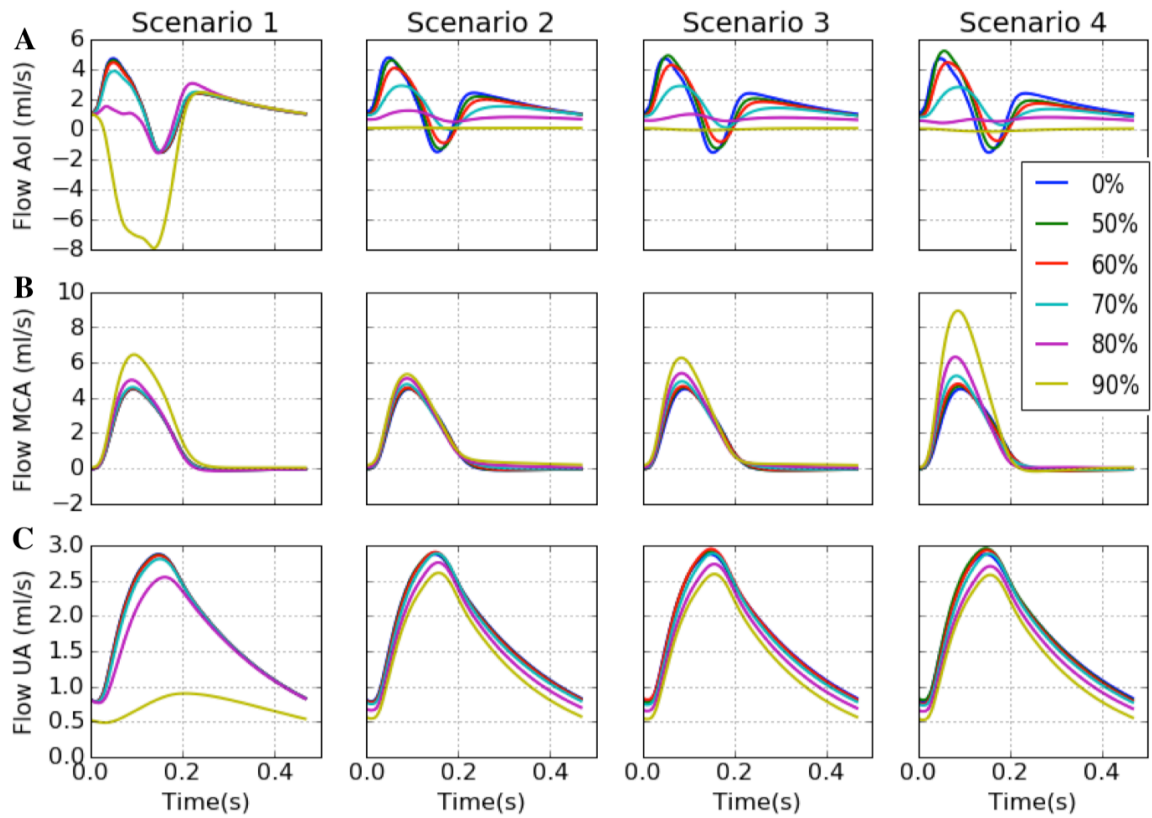

Fig 3. Blood flow through: aortic isthmus (AoI), middle cerebral artery (MCA) and umbilical artery (UA); for different percentages of stenosis. Scenario 1: coarctation of distal aortic isthmus; Scenario 2: hypoplasia of aortic isthmus; Scenario 3: hypoplasia of aortic isthmus and descending arch; Scenario 4: hypoplasia of aortic isthmus, descending arch and ascending arch.

The cerebro-placental ratio (Figure 4A) augmented with stenosis for all coarctation types; however, under distal isthmus coarctation the most severe increment was attained for $90 \%$ stenosis. The left ventricular pressure (Figure 4B) also increased with coarctation; it was observed that in aortic isthmus-arch hypoplasia (Scenario 4), when LV and RV output were similar the highest pressure was reached, at a non-physiological $90 \mathrm{mmHg}$ for $90 \%$ stenosis.

A

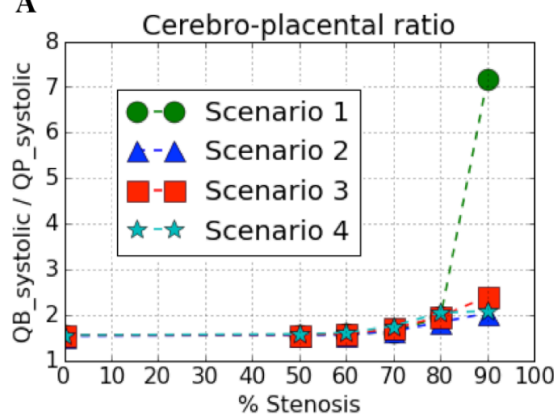

B

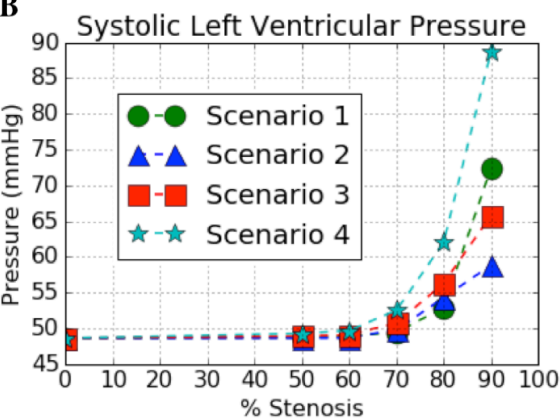

Fig 4. A. Cerebro-placental ratio. B. Left ventricular pressure; for different percentages of stenosis. Scenario 1: coarctation of distal aortic isthmus; Scenario 2: hypoplasia of aortic isthmus; Scenario 3: hypoplasia of aortic isthmus and descending arch; Scenario 4: hypoplasia of aortic isthmus, descending arch and ascending arch. 
The pressure drop over the coarctation for the four scenarios was plotted in Figure 5. It showed that pressure difference augmented with percentage of coarctation, in all the scenarios. Furthermore, the increase in pressure drop was more significant both, when the coarctation was located distal from the aorticductal junction and for the whole aortic arch hypoplasia.

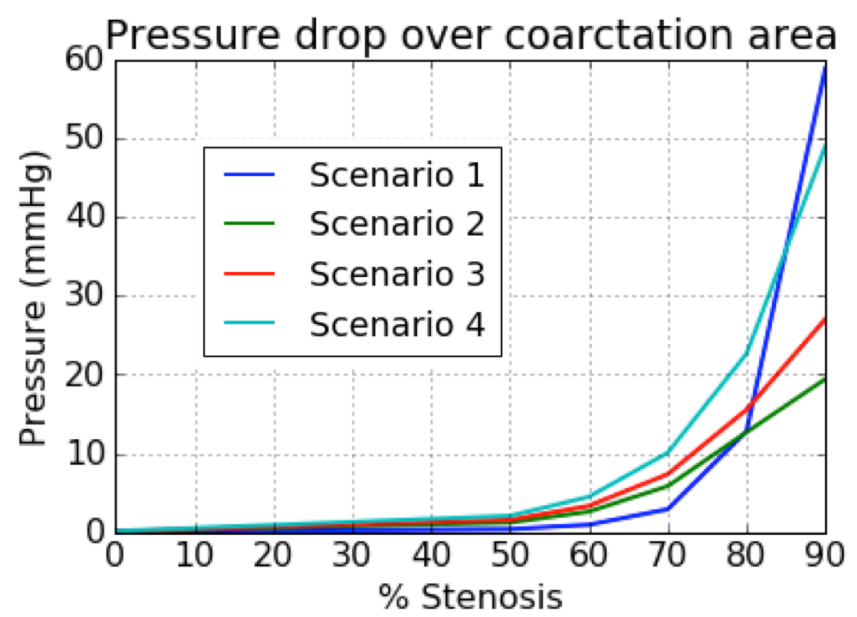

Fig 5. Maximum pressure drop over area of coarctation, against percentage of stenosis. Dark blue: coarctation of distal aortic isthmus; Green: hypoplasia of aortic isthmus, Red: hypoplasia of aortic isthmus and descending arch; Light blue: hypoplasia of aortic isthmus, descending arch and ascending arch.

\subsection{Modeling ventricular disproportion in aortic arch hypoplasia}

In clinical scenarios, the LV stroke volume (and size) decreases when the coarctation becomes more drastic. The driving force (as can be seen from Fig 4B) is likely the increase in LV pressure. Therefore we also simulated this RV/LV imbalance. One can see that reducing the amount of flow leaving the left ventricle favored the retrograde flow in the AoI, in aortic arch hypoplasia (Figure 6A); while increased stenosis resulted in a decrement of the AoI retrograde flow. Regarding the MCA blood flow (Figure 6B), it was increased by stenosis; nevertheless, under severe ventricular disproportion (20-80\%), MCA flow decreased with stenosis. Moreover, it was noticed that UA flow augmented with stenosis, under ventricular disproportion (Figure 6. C). The more severe the disproportion, the higher the increment with stenosis. 

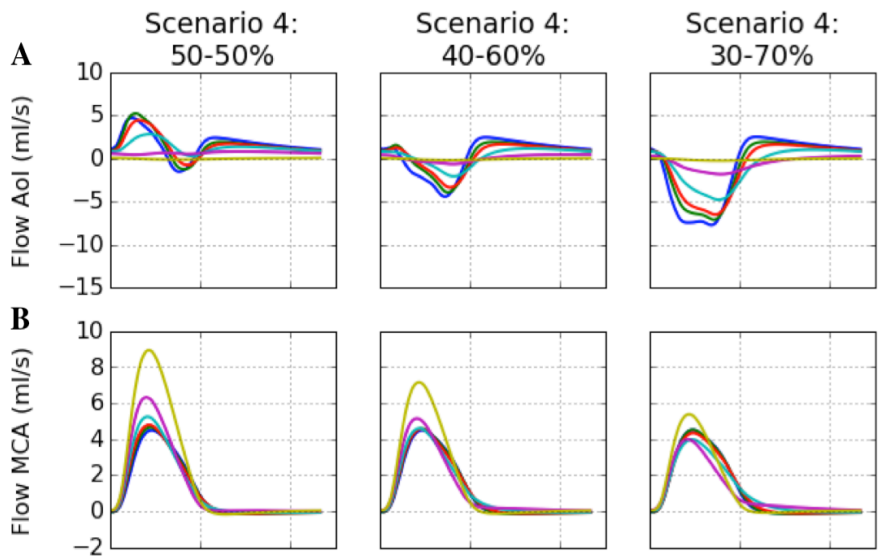

Scenario 4:
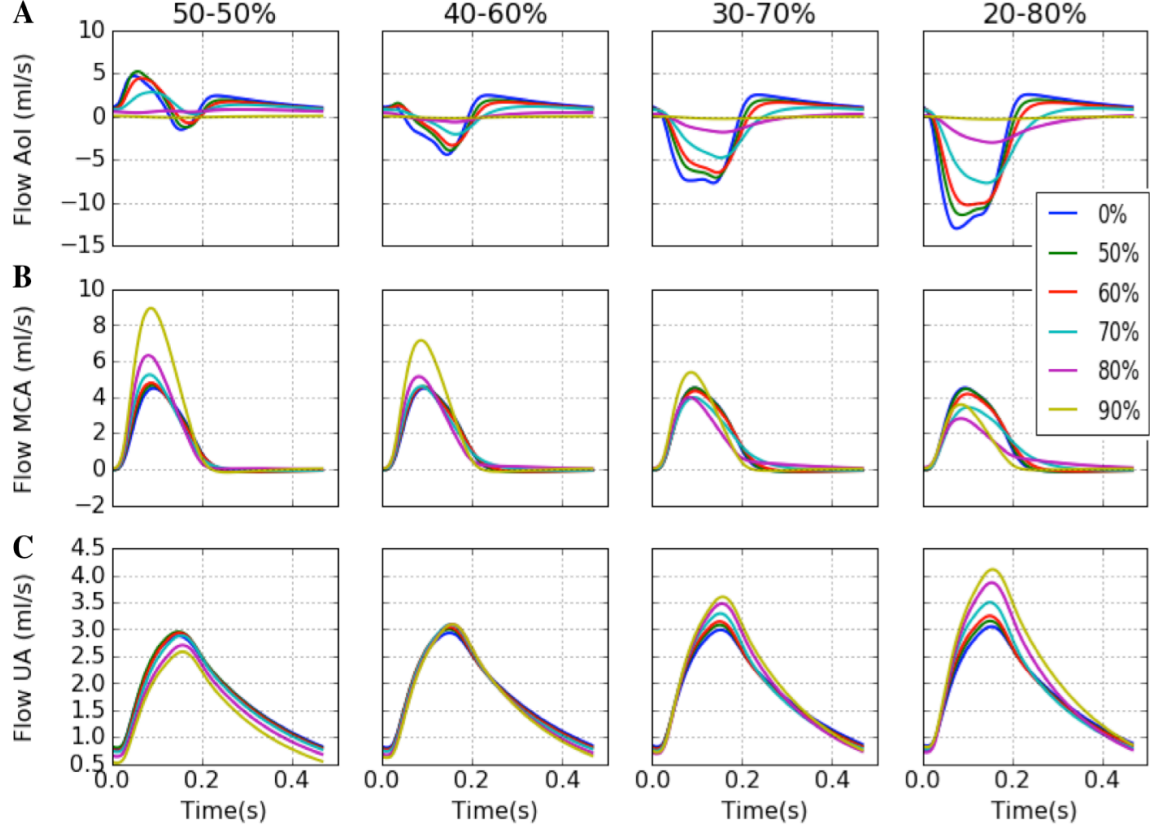

Fig 6. Blood flow through aortic isthmus (AoI), middle cerebral artery (MCA) and umbilical artery (UA), for different percentages of stenosis. Hypoplasia of aortic isthmus, descending arch and ascending arch, simulated for $50-50 \%, 40-60 \%, 30-70 \%$ and $20-80 \%$ proportion of left-right ventricle flow respectively.

Regarding cerebro-placental ratio (Figure 7A) it was observed to decrease with stenosis as ventricular disproportion became more severe. On the other hand, left ventricular pressure (Figure 7B) started to normalize. Ventricular disproportion favored the decrement of left ventricular pressure, also enhanced by coarctation.
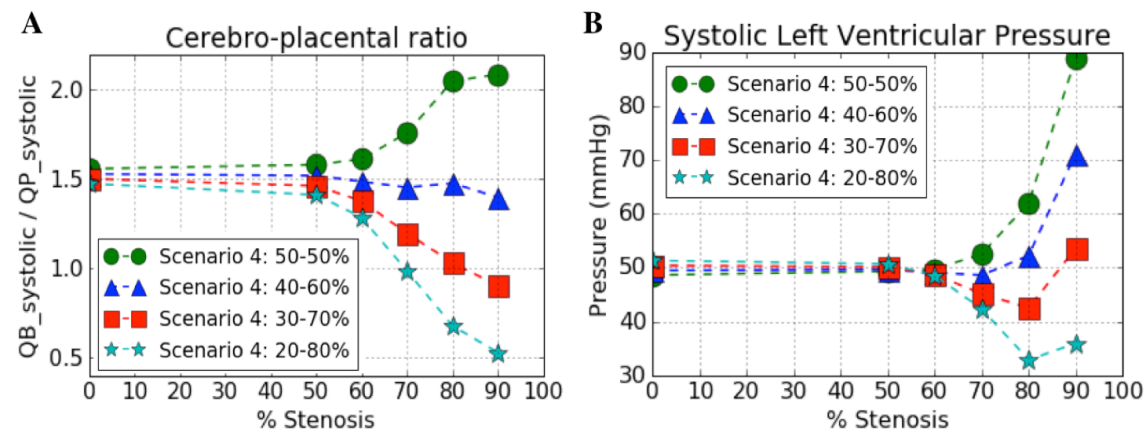

Fig 7. A. Cerebro-placental ratio. B. Left ventricular pressure; for different percentages of stenosis. Hypoplasia of aortic isthmus, descending arch and ascending arch, simulated for $50-50 \%, 40-60 \%, 30-70 \%$ and $20-80 \%$ proportion of left-right ventricle flow respectively. 


\section{Discussion}

We have successfully implemented a lumped model of the fetal circulation in CellML/OpenCOR and simulated the hemodynamic effects of different degrees and geometries of in-utero aortic coarctations.

Coarctation of the aorta is one of the most difficult cardiac defects to diagnose before birth. Antenatal diagnosis is crucial for early treatment and to reduce the risk of morbidity and mortality. For this purpose, we have implemented a patientspecific lumped model of the fetal circulation in OpenCOR, including the modeling of different types and degrees of aortic coarctation.

The results show that the current lumped model of the fetal circulation provides insight in the underlying mechanism of aortic coarctation. The reversal of the systolic AoI flow observed when coarctation is located distal from the aortic-ductal junction agrees with previous studies [1]. As a consequence, the blood to the brain increases while the blood flow to the lower body and placenta decreases. Since the coarctation is located just after the aortic-ductal junction, most of the blood coming through the ductus is redirected towards the upper body due to the narrowing of the distal aortic arch. The increase in MCA blood flow is also observed in the other scenarios, but more strongly in aortic isthmus and arch hypoplasia. Thus, as coarctation increases, blood from the ascending aorta can only reach the brachiocephalic trunk and just a small amount of blood passes through the narrowed arch towards the descending aorta. However, keeping RV/LV output balanced led to non-physiological LV pressures.

We also noticed that reducing the amount of outflow of the left ventricle, normalized the LV pressures and led to retrograde flow in the AoI, as supported by previous studies [12]. Nevertheless, when the whole aortic arch is hypoplastic (Scenario 4), less AoI retrograde flow is observed when increasing stenosis. Though it seems contradictory, MCA blood flow increases with stenosis, since the flow through the aortic arch is reduced and it favors blood flow towards the brachiocephalic trunk. This is again partly normalized when the relative amount of left ventricular outflow decreases up to $20 \%$ and the blood flow in the MCA is also reduced. On the other hand, blood flow in the UA increases with the amount of stenosis as well as when the difference between right and left relative amount of outflow rises, showing that more blood goes from the ductus arteriosus to the lower body and placenta.

Concerning the pressure drop over the narrowed area, we perceived that at around $80 \%$ stenosis the pressure gradient was around $20 \mathrm{mmHg}$. In current clinical practice, a coarctation (as measured postnatally) is considered significant from this value onwards, indicating that hemodynamic changes observed in our model agree with clinically established knowledge [13]. Therefore, the simulations provide more insights into the hemodynamics of aortic coarctation. Moreover this model provides patient-specific pressure gradient estimations, which are challenging to obtain in the clinic.

On the other hand, implementation of the model in CellML/OpenCOR reduces the computation time [10], making possible real time simulations, and it facilitates its reutilization for future applications.

The main limitation of this model is that fetal circulation is still quite simplified. However, future lines of work aim to overcome this limitation by modeling the heart, and thus, increasing resemblance to reality. Moreover, we haven't analyzed the effect of oxygen and vasoconstriction, which can also have an effect on the results. 


\section{Conclusion}

In this paper, we proposed the use of a lumped model of the fetal circulation to evaluate the hemodynamic changes in fetal aortic coarctation. Given the insight obtained, this tool might help to improve prenatal diagnosis of aortic coarctation.

\section{References:}

[1] A. Buyens, W. Gyselaers, A. Coumans, S. Al Nasiry, C. Willekes, D. Boshoff, et al. Difficult prenatal diagnosis: fetal coarctation. FVV in ObGyn. 4 (2012)

[2] J. Espinoza, R. Romero, J. P. Kusanovic, F. Gotsch, et al. Prenatal diagnosis of coarctation of the aorta with the multiplanar display and B-flow imaging using 4dimensional sonography. J Ultrasound Med. 28 (2009), 1375-1378. https://doi.org/10.7863/jum.2009.28.10.1375.

[3] R. Achiron, S. Zimand, Z. Rotstein, at al. Fetal aortic arch measurements between 14 and 38 weeks' gestation: in-utero ultrasonographic study. Ultrasound Obstet Gynecol, 15 (2000), 226-230. https://doi.org/10.1046/j.1469-0705.2000.00068.x

[4] H. Matsui, M. Mellander, M. Roughton, et al. Morphological and physiological predictors of fetal aortic coarctation. Circulation, 118 (2008) 1793-1801, https://doi.org/10.1161/CIRCULATIONAHA.108.787598.

[5] J. F. Kenny, T. Plappert, M. S. J. Sutton, et al. Changes in intracardiac blood flow velocities and right and left ventricular stroke volumes with gestational age in the normal human fetus: a prospective Doppler echocardiographic study. Circulation, 74 (1986), 12081216. https://doi.org/10.1161/01.CIR.74.6.1208

[6] A. Buyens, W. Gyselaers, A. Coumans, S. Al Nasiry, C. Willekes, D. Boshoff, I. Witters, et al. Difficult prenatal diagnosis: fetal coarctation. FVV in ObGyn. 4 (2012), 230-236.

[7] Y. Mukai, O. Samura, Y. Teraoka, \& M. Sasaki. Prenatal diagnosis of coarctation of the aorta using the three-vessel view by calculating the ratio of the diameter of the aortic root to that of the pulmonary artery. Ultrasound Obs. Gynecol. 44 (2014) 1793-1801, https://doi.org/10.1002/uog.14118.

[8]P. Garcia-Canadilla, P.A. Rudenick, F. Crispi, M. Cruz-Lemini, G. Palau, O. Camara, et al., A computational model of the fetal circulation to quantify blood redistribution in intrauterine growth restriction, PLoS Comput. Biol. 10 (2014) e1003667, http://dx.doi.org/10.1371/journal.pcbi.1003667.

[9] P. Garcia-Canadilla, P.A. Rudenick, F. Crispi, M. Cruz-Lemini, S. Triunfo, A. Nadal, et al., Patient-specific estimates of vascular and placental properties in growth-restricted fetuses based on a model of the fetal circulation, Placenta. 36 (2015) 981-989, http://dx.doi.org/10.1016/j.placenta.2015.07.130.

[10] P. Hunter. OpenCOR Tutorial (2016).

[11] M. Szpinda, Length growth of the various aortic segments in human foetuses. Folia Morphol. 67 (2008) 245-250.

[12] Y. Yamamoto, N. S. Khoo, P. A. Brooks, W. Savard, et al. Severe left heart obstruction with retrograde arch flow influences fetal cerebral and placental blood flow. Ultrasound Obstet Gynecol. 42 (2013), 294-299. http://dx.doi.org/10.1002/uog.12448.

[13] L. M. Beauchesne, H. M. Connolly, et al. Coarctation of the aorta: outcome of pregnancy. J Am Coll Cardiol, 38 (2001) 1728-1733. https://doi.org/10.1016/S07351097(01)01617-5. 
Acknowledgements. The work is supported by the European Union Horizon2020 research and innovation programme under grant agreement No 642676(CardioFunXion). The authors would like to thank the organizers of this project: Bart Bijnens and Mathieu De Craene. Finally, the authors would also like to thank David-Soto Iglesias for all the help provided with the conformal mapping of the endocardium. 